\title{
Observatorios urbanos en América Latina: ¿observar o participar?
}

\section{Urban observatories in Latin America: Observe or participate?}

\author{
Luis Miguel Valenzuela-Montes* \\ Juliana Carvalho-Cortes Silva**
}

\begin{abstract}
Resumen
El objetivo de esta investigación es avanzar en la comprensión del rol de los observatorios, específicamente en el contexto metropolitano latinoamericano, en lo que se refiere a su capacidad para promover la participación ciudadana utilizando Tecnologías de Información Geográfica (TIG). Se identificaron tipologías respecto al acceso y uso que hacen los ciudadanos de la información sobre fenómenos, políticas, planes y proyectos urbanos. La valoración realizada evidencia que actualmente el rol de estos observatorios, en general, no es precisamente proactivo ni interactivo, sino uno con limitada capacidad de promover la participación, la transparencia espacial y la toma de decisiones colectivas.
\end{abstract}

Palabras clave: Metrópolis latinoamericanas, Observatorios urbanos, Participación Tecnologías de la Información Geográfica.

\begin{abstract}
The goal of this paper is to advance in the understanding of the role of observatories, specifically in the Latin American metropolitan context, in terms of their ability to promote citizen participation using Geographic Information Technologies (GITs). The used methodology allowed us to identify different typologies regarding citizen access and use of the information about urban events, policies, plans and projects. The performed assessment makes it evident the fact that, in general, the role of these observatories is not exactly proactive or interactive, but one with limited ability to promote participation, spatial transparency and collective decision making.
\end{abstract}

Keywords: Latin American Metropolises, Urban Observatories, Participation, Geographic Information Technologies.

\footnotetext{
* Universidad de Granada, Espańa. Correo-e: lvmontes@ugr.es

** Pontificia Universidad Católica de Chile. Correo-e: jcarvalh@uc.cl
} 


\section{Introducción}

Los observatorios urbanos son entidades que cobran cada vez mayor importancia en temas relacionados con la gestión y la planificación de las metrópolis alrededor del mundo. En la actualidad, se institucionalizan progresivamente en respuesta a las demandas ciudadanas de más acceso a la información y de mayor transparencia en los procesos de toma de decisión; sin embargo, todavía no queda claro en qué medida estos observatorios influyen en los procesos de gestión y planificación de ciudades y territorios.

A pesar de que el término observatorio, en stricto senso, remite a un carácter más pasivo, caracterizando a un instrumento que posibilita hacer observaciones asociadas aquí principalmente con actividades de colección, sistematización y presentación de datos e informaciones, para el caso específico de los observatorios urbanos, esto no es necesariamente así, pues bastantes buscan, de forma declarada, interferir en los diversos procesos territoriales que observan, logrando, en ocasiones, operar como agentes activos y relevantes de los mismos.

En este sentido, los observatorios pueden hacer más que sólo observar (Repetti y Prélaz-Droux, 2003; Valenzuela y Soria, 2011; un-Habitat, 2014; Banco Mundial, 2014), pudiendo, por ejemplo, a través de la entrega de información a los ciudadanos, cooperar en la transparencia de los procesos de toma de decisión, así como en la construcción de escenarios futuros para las ciudades (Vajjhala, 2006).

No obstante, en el caso de América Latina, a pesar de su alto grado de urbanización, pareciera que la mayoría de sus observatorios urbanos han avanzado relativamente poco en esta dirección, particularmente, en lo que se refiere a entregar a la población posibilidades reales de participación en temáticas asociadas al territorio que habitan. Ante este panorama, y teniendo en cuenta que frente a la creciente complejidad de la dinámica espacial actual es extremamente importante reflexionar acerca de cuáles son las adaptaciones más críticas de la gobernanza metropolitana (Yeh, 2008; Fusero, 2009; Hernández-Moreno, 2009; Ganapati, 2011; Ferreira et al. 2012; Orellana, 2013) necesarias para la planificación urbana y territorial, se estableció, como objetivo central para este estudio, analizar en qué medida los observatorios urbanos son en Latinoamérica instrumentos operativos y participativos para la planificación metropolitana.

Para llevar a cabo este propósito, la metodología se inspiró en la de Valenzuela y Soria (2011), que establece una serie de criterios cualitativos para evaluar el nivel de influencia que tienen los observatorios territoriales y urbanos europeos en el proceso de planificación. Estos criterios fueron adaptados y ampliados para los propósitos específicos de esta in- 
vestigación, de modo que, por un lado, incorporan la variable de la participación ciudadana y, por otro, centran el análisis en estudios de caso que hacen uso de Tecnologías de la Información Geográfica (TIG).

La decisión de incorporar estos aspectos en la metodología se fundamenta en la idea de que los habitantes de una urbe son agentes centrales en sus dinámicas y que deberían disponer de información confiable, actual y espacial sobre su ciudad para tomar sus decisiones, tanto en el corto como en el mediano y largo plazo (Hendriks y Vriens, 2000; Kingston et al., 2000; Carsjens y Ligtenberg, 2007; González et al., 2008; Bailey y Grossardt, 2009; McHugh et al., 2009; Ganapati, 2011; Mansourian et al., 2011). Considerando esta fundamentación, se asume como premisa que "la habilidad de los individuos y grupos sociales de mapear, en un sentido verdaderamente geográfico, su visión de futuro es un prerrequisito para una planificación informada y sustentable" (Carver, 2003: 64).

Además, respecto a la participación ciudadana en temas de planificación urbana, las TIG pueden, particularmente a través de internet, viabilizar la distribución y visualización de información en su expresión territorial (Kearns et al., 2003; Rojas-Caldelas y Corona-Zambrano, 2008), acompañada de un acceso equitativo ininterrumpido (Duncan y Lach, 2006; Fusero, 2009). Éstas también pueden promover la transparencia de diversas políticas públicas urbanas, informando, por ejemplo, respecto a dónde se ubicará un servicio sanitario o cuál será el trazado de una nueva línea de metro, asimismo, debido al flujo de información que generan podrían enriquecerse las decisiones y proyectos en torno a las acciones urbanas (Haque, 2001; Hansen y Prosperi, 2005; McCall y Dunn, 2012).

En este contexto las TIG y los observatorios urbanos pueden contribuir no sólo a la incorporación relevante de la ciudadanía a las políticas públicas urbanas sino también al empoderamiento de la comunidad mediante su concienciación y conocimiento urbanos (Hanzl, 2007; McHugh et al., 2009).

Desde esta perspectiva, se pueden destacar tres potenciales ventajas de la participación en red:

(i) no está restringida por la ubicación geográfica y, por lo tanto, no limita la posibilidad de participación a aquellas personas que pueden estar presentes en un lugar específico;

(ii) está disponible a cualquier hora del día y cualquier día de la semana, lo que significa que cada uno puede decidir participar cuando le parezca más conveniente, disminuyendo los riesgos de no participación debido a incompatibilidades de agendas; 
(iii) y es menos confrontacional, disminuyendo los riesgos de no participación asociados a factores psicológicos (Kingston et al., 2000; Hérnandez-Moreno, 2009).

La apertura de canales de participación a través de la web puede generar mayor confianza en el gobierno por parte de individuos y comunidades, aumentar el involucramiento ciudadano en asuntos públicos, o bien, legitimar programas y políticas, entre otros aspectos (Haque, 2001; McCall y Dunn, 2012), promoviendo así posibles iniciativas de e-government (Ganapati, 2011). Uno de los principales instrumentos disponibles a los gobiernos para entregar información a los ciudadanos son los Sistemas de Información Espacial, que, de acuerdo con Fusero (2009), fueron creados para ordenar datos e integrar informaciones que de otro modo quedarían dispersas y desconectas, ya que son distintos y diversos lo entes y agencias responsables por su recolección, análisis, almacenamiento y publicación.

Los observatorios urbanos muchas veces cumplen esta función, ocupando espacios en el espectro de intercambio de información entre sociedad y gobierno que muchas veces son débiles o incluso inexistentes. En el contexto actual, caracterizado por una amplia y creciente diseminación de las TIG, es importante analizar cómo se puede explorar el potencial de estas tecnologías para generar nuevas instancias de participación ciudadana, particularmente en lo que se refiere a la planificación urbana (Álvarez-Lobato, et al., 2007; Graham, 2000).

La selección de los observatorios se basó en una serie de condiciones básicas, empezando por la ubicación geográfica y la escala, lo que significó observatorios urbanos (i) latinoamericanos que (ii) se enfocan en áreas metropolitanas. Además, estos observatorios tenían que: (i) ser públicos, contando con páginas web accesibles a través del uso de computadoras o celulares que cuenten con conexión a internet; (ii) hacer uso de las TIG, a través, por ejemplo, de Sistemas de Información Geográficos Participativos, Web Mapping o cartografía digitalizada, y (iii) tratar sobre temas asociados con la planificación del territorio. Estos criterios, además de hacer factible una investigación a distancia de los observatorios a través de su presencia online, reflejan, en su conjunto, una apuesta por el potencial de las TIG como una herramienta para la participación ciudadana en temas de planificación urbana (Dennis, 2006; González et al., 2008; Bailey y Grossardt, 2009).

Con base en estos lineamientos se hizo una primera selección de 16 observatorios, de seis países (Argentina, Brasil, Chile, Colombia, Perú y Uruguay). Estos fueron sometidos, a su vez, a un conjunto de criterios de valoración, vinculados sobre todo con la intención de explorar y cribar 
aquellos casos que mejor permitan evaluar su capacidad efectiva de establecerse como instrumentos operativos y participativos para la planificación metropolitana. Como resultado de este filtro metodológico, siete observatorios fueron seleccionados (de Argentina, Brasil, Chile y Perú).

Esta segunda identificación de casos fue examinada en mayor detalle, siendo estos nuevamente sometidos a criterios cualitativos de análisis, pero con el objetivo específico de evaluar dónde se ubican en el espectro que va desde la categoría de entidades más pasivas -dedicadas mayoritariamente a observar procesos y dinámicas que se dan en el territorio y generar información acerca de ellos- hasta la mayor operatividad y participación en la planificación.

Los resultados encontrados apuntan a un panorama en el cual los observatorios tienden a ser más bien pasivos y raras veces logran superar el nivel informativo respecto a la incorporación de la ciudadanía (Huerta, 2013). Son escasas las prácticas de capacitación y educación (Freire y Villar, 2010), incipientes las de cooperación y promoción de buenas prácticas y casi inexistentes las de generación de escenarios futuros (Elwood, 2006; Davoudi y Strange, 2009). Derivadas de estos resultados, las conclusiones tratan, por un lado, de una reflexión acerca del potencial que tienen estos observatorios para los temas de planificación y gestión, particularmente a través del uso de las Tig en el marco de la Sociedad en red. Y, por otro lado, se presentan algunas claves para que el diseño de observatorios sea más operativo, o sea, proactivo, en lo que se refiere a la participación ciudadana, fomentando así líneas de investigación que propicien observatorios que vayan más allá de sólo observar.

\section{Metodología}

La metodología utilizada en este estudio fue generada a partir de la integración de, por un lado, el modelo propuesto por Valenzuela y Soria (2011) para el análisis de observatorios urbanos y territoriales en Europa y, por otro, asumiendo el esquema conceptual construido por Arnstein (1969) acerca de los niveles de participación ciudadana en temas de planificación, también conocido como escalera de participación, como referente teórico (complementado o actualizado por Nogué et al., 2010). De la integración tanto del modelo como del fundamento conceptual surge la metodología aplicada (Carvalho, 2013) desarrollada a continuación.

La primera etapa, que consistió en selección de la muestra inicial de casos para el análisis, fue realizada de forma circunstancial, a través de la navegación en la internet. El norte de tal búsqueda estaba establecido por el propósito de seleccionar un conjunto de observatorios urbanos que fuera representativo de la temática identificada en el contexto latinoameri- 
cano. Como parámetros de búsqueda, además de preestablecer páginas que estuvieran en castellano o portugués, algunos conceptos y expresiones fueron claves, como participación ciudadana, planificación y "observatorio urbano" (específicamente entre comillas para evitar otros tipos de observatorios, como los astronómicos).

De los resultados obtenidos en las búsquedas iniciales, se visitaron las páginas de aquellos observatorios relacionados con la escala metropolitana y se seleccionaron los que hacían uso de alguna Tecnología de Información Geográfica para entregar información. Vale destacar que inicialmente se consideró la posibilidad de utilizar como grupo inicial todos los observatorios latinoamericanos que forman parte de la red de observatorios urbanos locales de la UN-Habitat. Sin embargo, el listado disponible en la página web de esta organización internacional data del 2002 y contempla ciudades de distintas escalas socioespaciales, lo que dificultaría la comparación entre los casos. Además, no todos los observatorios de este grupo cuentan con plataformas electrónicas disponibles en la internet y muy pocos entregan información geográfica.

Sin embargo, los objetivos establecidos en la estrategia operativa de esta red (UN-Habitat, 2014) son asumidos en este trabajo como referencias teóricas para la caracterización de un observatorio urbano. Con base en estas consideraciones, los criterios específicos de selección, que caracterizaron y clasificaron esta exploración inicial en búsqueda de observatorios urbanos latinoamericanos, fueron los siguientes:

- ser de carácter público, disponible online (no necesariamente operante hasta la fecha), accesible a través del uso de computadoras o celulares que cuenten con conexión de internet;

- tener relación con temas de planificación urbana y estar relacionado a una metrópolis o área metropolitana (más que 4,5 millones de habitantes);

- hacer uso de las Tig, a través, por ejemplo, de Sistemas de Información Geográficos Participativos, Web Mapping o Cartografía digitalizada, para entregar información de forma gratuita.

La aplicación de estos criterios básicos evaluó en una primera fase selectiva un conjunto de 16 observatorios urbanos que están listados en el cuadro 1. Algunos de ellos no tenían foco en una urbe específica (se puede citar como ejemplo el caso del Observatorio de las Metrópolis, de Brasil, que abarca 14 metrópolis y una aglomeración urbana en su ámbito de trabajo). En estos casos, se optó por designar una metrópolis específica para cada ámbito, obedeciendo, para este proceso de criba, el énfasis dado por los observatorios mismos a las ciudades observadas. Cabe 
resaltar que todas las metrópolis presentes en la selección son representativas de sus países y objeto de trabajos comparativos relevantes de la bibliografía latinoamericana sobre metropolización, desarrollo urbano, planificación y ciudad, en general (Cicolella, 2006; Portes et al., 2008).

\section{Cuadro 1}

Selección de casos metropolitanos latinoamericanos

\begin{tabular}{lll}
\hline \multicolumn{1}{c}{ Nombre } & \multicolumn{1}{c}{ Metrópolis } & \multicolumn{1}{c}{$\begin{array}{c}\text { Tipo de visualización de } \\
\text { datos espaciales disponible }\end{array}$} \\
\hline Mapa Interactivo de Buenos Aires & Buenos Aires & Mapa interactivo, sig \\
Observatorio Urbano Local & Buenos Aires & Cartografía digitalizada \\
Observatorio de Resultados de Gobierno & Buenos Aires & Mapa interactivo \\
Observatorio do Milenio & Belo Horizonte & Cartografía digitalizada \\
Rio Como Vamos & Rio de Janeiro & Mapa interactivo \\
Rede Nossa São Paulo & São Paulo & Cartografía digitalizada \\
Observatório das Metrópolis & Rio de Janeiro & Cartografía digitalizada \\
Observatorio Urbano & Lima & Mapa interactivo \\
Observatorio Urbano Perú & Lima & Mapa interactivo \\
Observatorio de Montevideo & Montevideo & Mapa interactivo, sig \\
CAS - Climate Adaptation Santiago & Santiago & Mapa interactivo, sig \\
Suma Verde & Santiago & Mapa interactivo \\
Observatorio de Ciudades Uc & Santiago & Cartografía digitalizada \\
Observatorio Urbano MINVU & Santiago & Mapa interactivo \\
SIGUE & Bogotá & Mapa interactivo \\
Red de Ciudades Cómo Vamos & Bogotá & Cartografía digitalizada \\
\hline
\end{tabular}

Fuente: Carvalho (2013).

Este primer conjunto de 16 observatorios fue sometido a un segundo grupo de criterios, con el objetivo de refinar la muestra inicial ${ }^{1}$ a casos en los cuales no solamente la planificación estuviera presente, sino que además, existiera una preocupación por la promoción de la participación ciudadana informacional en el marco de la sociedad en red (Castells, 1995) y una mirada más integral sobre el territorio, reflejando un interés en contemplar la complejidad de las metrópolis.

Siguiendo este razonamiento, se consideraron como referencia para el desarrollo de los criterios, los Objetivos de la Cumbre Mundial Sobre la Sociedad de la Información, también conocida como la Agenda de

\footnotetext{
${ }^{1}$ Téngase en cuenta que fue suficiente que un caso tuviera una apreciación "nula" o "baja" en alguno de los criterios para que fue excluido del conjunto a ser analizado.
} 
Túnez para la Sociedad de la Información, así como los Objetivos de la Agenda 21, ambos de las Naciones Unidas. Los primeros fueron atendidos por representar en el vínculo entre personas y tecnologías, estando enfocados en la democratización del acceso y del uso de las tecnologías. Fueron acordados por los representantes de los países en el marco de la Unión Internacional de Telecomunicaciones y figuran como los principios comunes a nivel internacional en lo que se refiere a un desarrollo inclusivo de la Sociedad en Red, en el cual las tecnologías desempeñan un papel central en la formulación e implementación de políticas públicas (ITU, 2006).

En cuanto a los segundos, los Objetivos de la Agenda 21, representan el vínculo entre ciudadanía y ámbitos locales. Mientras los Objetivos del Milenio están más enfocados en la escala macro (regional o nacional), la Agenda 21 tiene un fuerte acercamiento a la escala local, que pretende acercar a los habitantes a sus ciudades, mediante el impulso estratégico de la participación ciudadana generada por la información espacial y ambiental de los observatorios urbanos. En estos objetivos el desarrollo sustentable es una meta transversal a las todas las temáticas tratadas (UN, 2001).

Vale destacar que los Objetivos del Milenio y la Agenda 21, conjuntamente con la Agenda Hábitat están estrechamente relacionados con el programa de observatorios urbanos de la UN-Habitat, el cual está conformado por tres escalas principales: urbana o local, regional y nacional. Complementariamente, a la escala internacional está el Observatorio Urbano Mundial, responsable de coordinar los observatorios que participan en esta red (Ferreira et al., 2012; un-Habitat, 2014). Sin embargo, no todos los observatorios existentes que participan de esta red cuentan con presencia en internet y otros que si están en la web no hacen uso de las TIG, no cumpliendo con los parámetros establecidos en este diseño metodológico.

Complementariamente a los criterios de coherencia con estos objetivos se suman otros dos (cuadro 2). Uno enfocado en la relación de los observatorios con la planificación urbana y, otro, relacionado con el carácter transversal de las temáticas abarcadas por los observatorios, excluyendo, en consecuencia, a aquellos que se dedican exclusivamente a un ámbito urbano, ya que se alejan de la perspectiva metropolitana, interactiva, abierta y transversal que caracteriza al enfoque de esta investigación sobre los observatorios, como promotores de la participación y la transparencia sobre la toma de decisiones de la planificación urbana. Por lo tanto, en su conjunto los criterios cumplen el rol de seleccionar casos comparables en los cuales, además de haber un interés en observar la metrópolis, exis- 
ta también una preocupación por colaborar y promover la participación en su planificación.

A continuación se enumeran y esquematizan estos criterios en el (cuadro 2), presentando los parámetros de evaluación asociados a cada uno de ellos, constituyendo una aportación del proceso metodológico aplicado en esta investigación. Para cada uno de los criterios existen tres niveles de valoración: nula, media y alta. En el caso de recibir una valoración nula, el observatorio quedará excluido del proceso de análisis de potencial de incorporación ciudadana.

\section{Cuadro 2 \\ Criterios de selección de casos y su valoración cualitativa}

\begin{tabular}{|c|c|c|}
\hline \multicolumn{3}{|c|}{ Valoración cualitativa de los criterios de selección de los casos } \\
\hline Criterios de selección & Parámetros a evaluar en cada criterio de selección & $\begin{array}{l}\text { Valoración } \\
\text { cualitativa }\end{array}$ \\
\hline Criterio 1 & $\begin{array}{l}\text { 1. No adopta ni difunde los objetivos sociales } \\
\text { derivados de la Cumbre, enfocados en promover } \\
\text { una Sociedad de la Información más inclusiva. }\end{array}$ & 1. Nula \\
\hline \multirow{2}{*}{$\begin{array}{l}\text { Coherencia con los } \\
\text { Objetivos de la Cum- } \\
\text { bre Mundial Sobre la } \\
\text { Sociedad de la Infor- } \\
\text { mación }\end{array}$} & $\begin{array}{l}\text { 2. Busca involucrar cada vez más personas, } \\
\text { promoviendo principalmente el acceso a infor- } \\
\text { mación a través de las tecnologías. }\end{array}$ & 2. Media \\
\hline & $\begin{array}{l}\text { 3. Busca involucrar cada vez a más personas, } \\
\text { promoviendo no sólo el acceso a información a } \\
\text { través de las tecnologías sino también la capaci- } \\
\text { tación. }\end{array}$ & 3. Alta \\
\hline Criterio 2 & $\begin{array}{l}\text { 1. No adopta ni difunde los objetivos territoria- } \\
\text { les derivados de la Agenda } 21 \text {. }\end{array}$ & 1. Baja \\
\hline \multirow[t]{2}{*}{$\begin{array}{l}\text { Coherencia con los ob- } \\
\text { jetivos de la Agenda } 21\end{array}$} & $\begin{array}{l}\text { 2. Busca cooperar con la promoción de un de- } \\
\text { sarrollo sostenible. }\end{array}$ & 2. Media \\
\hline & $\begin{array}{l}\text { 3. Busca promover un desarrollo sostenible con } \\
\text { participación ciudadana. }\end{array}$ & 3. Alta \\
\hline Criterio 3 & 1. No se encuentra entre sus objetivos. & 1. Nula \\
\hline \multirow[t]{2}{*}{$\begin{array}{l}\text { Vínculos con la plani- } \\
\text { ficación urbana }\end{array}$} & $\begin{array}{l}\text { 2. Se encuentra entre sus objetivos realizar se- } \\
\text { guimiento del proceso de planificación sin in- } \\
\text { tervenir en el mismo. }\end{array}$ & 2. Media \\
\hline & $\begin{array}{l}\text { 3. Se encuentra en sus objetivos intervenir de } \\
\text { forma directa en la planificación urbana. }\end{array}$ & 3. Alta \\
\hline Criterio 4 & $\begin{array}{l}\text { 1. Se centra específicamente en un aspecto im- } \\
\text { plicado en la planificación. }\end{array}$ & 1. Baja \\
\hline \multirow[t]{2}{*}{$\begin{array}{l}\text { Carácter transversal- } \\
\text { territorial }\end{array}$} & $\begin{array}{l}\text { 2. Se centra en la planificación, aunque priori- } \\
\text { zando una temática específica. }\end{array}$ & 2. Media \\
\hline & $\begin{array}{l}\text { 3. Asume una perspectiva transversal sobre las } \\
\text { temáticas urbanas. }\end{array}$ & 3. Alta \\
\hline
\end{tabular}

Fuente: basado en Valenzuela y Soria (2011). 
El resultado de la aplicación de estos criterios a los 16 casos seleccionados se encuentra sintetizado en el cuadro 3, e indicando caso por caso las valoraciones asignadas a cada uno de ellos en cada categoría:

\section{Cuadro 3}

\section{Resultados de la aplicación de los criterios de selección a los casos de estudio seleccionados}

Resultado de la aplicación de los criterios de selección a los casos sudamericanos y urbanos Caso de estudio Criterio 1 Criterio 2 Criterio 3 Criterio 4

Seleccionados para el análisis

Observatorio Urbano Local (Buenos Aires)

Observatorio de Resultados de Gobierno (Buenos Aires)

Observatório das Metrópolis (Rio de Janei-

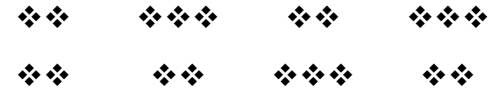
ro)

Observatorio Urbano Perú (Lima)

Observatorio Urbano (Lima)

Observatorio de Ciudades uc (Santiago)

Observatorio Urbano MINVU (Santiago)

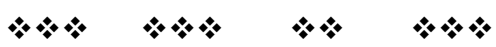

No seleccionados para el análisis

Mapa Interactivo de Buenos Aires (Buenos Aires)

Observatorio do Milenio (Belo Horizonte)

Rio Como Vamos (Rio de Janeiro)

Rede Nossa São Paulo (São Paulo)

Observatorio de Montevideo (Montevideo)

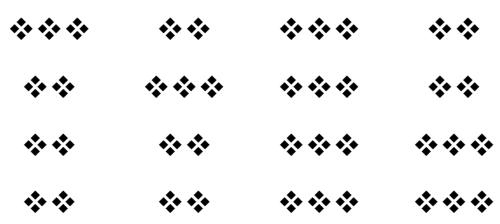

CAS Climate Adaptation Santiago (Santiago)

Suma Verde (Santiago)

SIGUE

Red de Ciudades Cómo Vamos

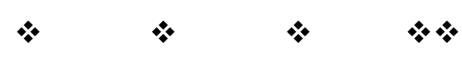

Observación: simboliza valoración nula o baja, simboliza media y $*$ simboliza alta. Fuente: Carvalho (2013).

De esta forma, fueron siete los casos seleccionados para la etapa de análisis del estudio, de cuatro países latinoamericanos, a saber: Argentina, Brasil, Chile y Perú. En esta etapa se evaluó el tipo de incorporación 
ciudadana que promueven estos observatorios a través de sus respectivos sitios web, particularmente, a través del uso de las Tig.

Es importante hacer notar el énfasis puesto en el asunto de la participación ciudadana a la hora de valorar en qué medida estos observatorios influyen en los procesos de planificación metropolitana. La opción o el interés por esta dimensión se debe al entendimiento de que los observatorios tienen un potencial intrínseco de operar en pro de la transparencia de procesos decisorios (Kingston et al., 2000, Song y Sun, 2010; Vajhala, 2006; Mansourian et al., 2011; McCall y Dunn, 2012), en la medida que las prácticas de colección, sistematización y presentación de datos e informaciones - prácticas que son, al fin y al cabo, un denominador común entre los observatorios- generan informaciones y resultados públicos. Estos resultados, a su vez, pueden informar a la ciudadanía y, potencialmente, incentivar su participación (Duncan y Lach, 2006; Dennis, 2006, Carsjens y Ligtenberg, 2007).

Metodológicamente, el análisis de las posibilidades de "incorporación ciudadana" planteado por estos observatorios en sus plataformas web fue realizado en base al esquema conceptual propuesto por Arnstein (1969), más conocido como la escalera de la participación. De acuerdo con este autor, la atención debe estar puesta en la distribución de poder existente en cada uno de los niveles de participación. A continuación se ilustra una versión adaptada de esta estructura de niveles, asociando a cada uno de los tres niveles principales características y prácticas que debieran existir. Vale notar que esta adaptación es fidedigna al marco conceptual del autor y fue realizada tomando en consideración los aportes del Observatorio del Paisaje de Cataluña (Nogué et al., 2010).

Fue tomando como referencia esta tipología de niveles de participación (cuadro 4), en el que se establecieron los criterios de evaluación que permiten examinar qué posibilidades de incorporación ciudadana presenta cada uno de estos observatorios a los individuos. El diseño de este grupo de criterios sigue la lógica de evaluación gradual plateada por Valenzuela y Soria (2011), lo que implica que hay una obligatoriedad en cumplir con al menos un criterio de un nivel específico para que sea contabilizado un criterio de asociado a un nivel superior a éste. Así, por ejemplo, si un caso cumple con el criterio J, asociado al nivel 3, pero no cumple con ninguno de los criterios del nivel 2 , se considera que posibilita una participación débil (o incluso una no-participación, a través, por ejemplo, de una incorporación de carácter manipulador). 


\section{Cuadro 4 \\ Niveles de participación ciudadana}

Estructura de los niveles definidos y ejemplos de las posibilidades encontradas en cada uno de ellos para la incorporación de la ciudadanía en procesos de planificación territorial

Participación fuerte o Menos control sobre el proceso por parte de aquellos que lo transformadora promueven.

Los participantes tienen más influencia sobre los resultados.

Requiere una explicación acerca de la relevancia de la participación.

Tiene un efecto transformativo en los participantes.

Posibilita el trabajo colaborativo y las decisiones colectivas.

Participación débil o Más control sobre el proceso por parte de aquellos que lo proconsulta pública mueven.

Poca capacidad de la sociedad civil para influenciar los resultados.

No se requiere una explicación acerca de la relevancia de la participación.

Individuos entregan información y hacen demandas.

Ciudadanos no participan en la toma de decisiones.

No-participación o Poco o ningún control sobre el procesos que se desarrollan.

acceso a información Poco o ningún espacio para contestación.

Se promueve incorporación bajo la condición que favorezca el proceso.

Gran distancia entre tomadores de decisión y participantes.

Los participantes sólo reciben información, flujo unilateral.

Fuente: elaboración propia con base en el trabajo de Arnstein (1969) y el Observatorio del Paisaje de Cataluña (2010).

Los criterios de evaluación derivados de esta estructura de niveles de participación han sido detallados en el cuadro 5. Los resultados de la aplicación de estos criterios y el análisis de estos resultados son presentados en la próxima sección de este documento, pero antes de adentrarse en esa etapa es importante explicar algunas características claves de estos criterios.

Como en este trabajo se utilizan las páginas web de los observatorios como material de análisis, los criterios establecidos para identificar las posibilidades de participación ciudadana que cada uno de los observatorios genera reflejan una preocupación central con el flujo de información. Siguiendo la proposición de Arnstein (1969) y los parámetros del Observatorio de Paisaje de Cataluña (2010), se considera que mientras mayores sean las posibilidades de flujos bidireccionales de información, mayores son las posibilidades de participación de individuos y comuni- 


\section{Cuadro 5 \\ Criterios para identificar las posibilidades de participación viabilizadas en cada caso}

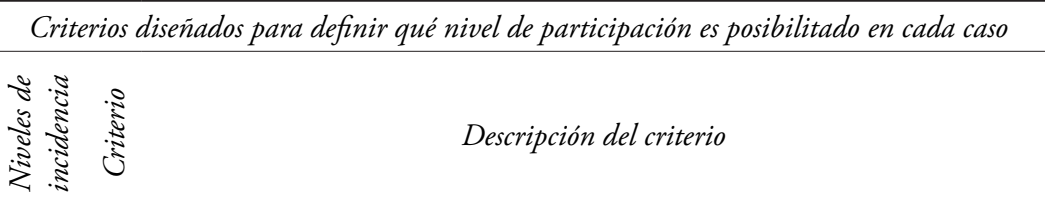

Nivel 1 - no-participación o acceso a información
A Entrega información territorial de forma gratuita.
B Permite la visualización interactiva de información espacial/georreferenciada.
C Realiza la elaboración propia de información y cartografía, y las publica.

Nivel 2 - participación débil o consulta publica

D Permite que individuos suban datos e información relacionados al territorio.

E Es respaldado por una ley de transparencia nacional.

F Realiza encuestas acerca de temáticas relacionadas a la planificación urbana.

G Establece espacios de discusión, como foros online.

Nivel 3 - participación fuerte o transformadora

$\mathrm{H}$ Establecer elementos que garanticen el accountability de los procesos territoriales.

I Posibilita la contestación de propuestas y/o la construcción de alternativas espaciales.

J Promueve actividades de educación y/o capacitación en el uso de las TIG.

K Garantiza feedback a las demandas ciudadanas.

Fuente: Carvalho (2013).

dades en procesos de toma de decisión y de planificación urbana, es por eso que en los criterios agrupados en el nivel 1 el flujo es estrictamente unidireccional.

Son aspectos de entrega de información (Repetti y Prélaz-Droux, 2003) que no contemplan ningún mecanismo para verificar la actualidad o veracidad de la información, lo que explica la posibilidad de manipulación asociada a este nivel de participación. Es también por eso que las leyes de transparencia comparecen en el nivel dos, haciendo un hincapié importante para garantizar la confiabilidad de la información. En este nivel también aparecen los criterios más débiles de bidireccionalidad de flujo, relacionados con la subida de información por parte de los usuarios de la página y las encuestas que pueden existir en estas plataformas.

Además está el criterio asociado al recurso de formación de redes, ya que los usuarios pueden relacionarse y conocerse en los foros de discusión. 
En el tercer y último nivel están los criterios asociados a la bidirecionalidad fuerte, por así decirlo, de flujos de información, porque involucran una retroalimentación entre usuarios y plataforma, estableciendo flujos potencialmente continuos y abriendo espacios para la proposición de alternativas (McCall y Dunn, 2012). Complementariamente, el criterio de educación y capacitación responde a una necesidad de promover la comprensión de los contenidos abordados, la habilidad de uso de las herramientas disponibles y la capacidad de analizar y reflexionar las informaciones intercambiadas.

En este sentido, se considera que cuanto mayor es la posibilidad de generar flujos bidireccionales de información, es más probable la incorporación ciudadana generada por los observatorios a través de sus páginas web (Mansourian et al., 2011). Además, tal como se mencionó anteriormente, se asume que es de suma importancia que los ciudadanos tengan acceso a la expresión territorial de la información sobre sus ciudades para promover una planificación responsable y sustentable, lo que explica la fuerte presencia de aspectos geográficos y espaciales en la construcción de los criterios.

\section{Resultados y discusión}

Los siete casos de observatorios metropolitanos seleccionados para la etapa de análisis de este estudio presentan una serie de características en común; sin embargo, a pesar de estas similitudes, cada uno de ellos tiene sus particularidades, de forma que aquellas consideradas más relevantes para el análisis han sido sintetizadas en el cuadro 6.

Los observatorios también difieren en relación con las posibilidades de participación que entregan a los ciudadanos, tal como se puede observar en el cuadro que sigue (cuadro 7), que esquematiza y compendia los resultados de la aplicación de los criterios de evaluación.

De los siete casos seleccionados, sólo uno alcanza el nivel 3 de participación, el Observatorio de las Metrópolis; sin embargo, hay que asumir este resultado con cautela, pues este observatorio logra alcanzar este nivel por ser uno de carácter más académico, fuertemente vinculado a instituciones de investigación e interesado en la realización de estudios comparables entre grandes metrópolis brasileñas. Esto ayuda a explicar porque cumple justamente con el criterio J, enfocado en la educación y capacitación, único criterio que lo califica en el nivel superior. Esto se debe al hecho que su funcionamiento depende de una red de docentes, investigadores y estudiantes, $y$, como corolario, tiene una serie de programas de formación educacional en diversos niveles: graduación, posgrado, cursos de capacitación y educación a distancia a él asociados. 


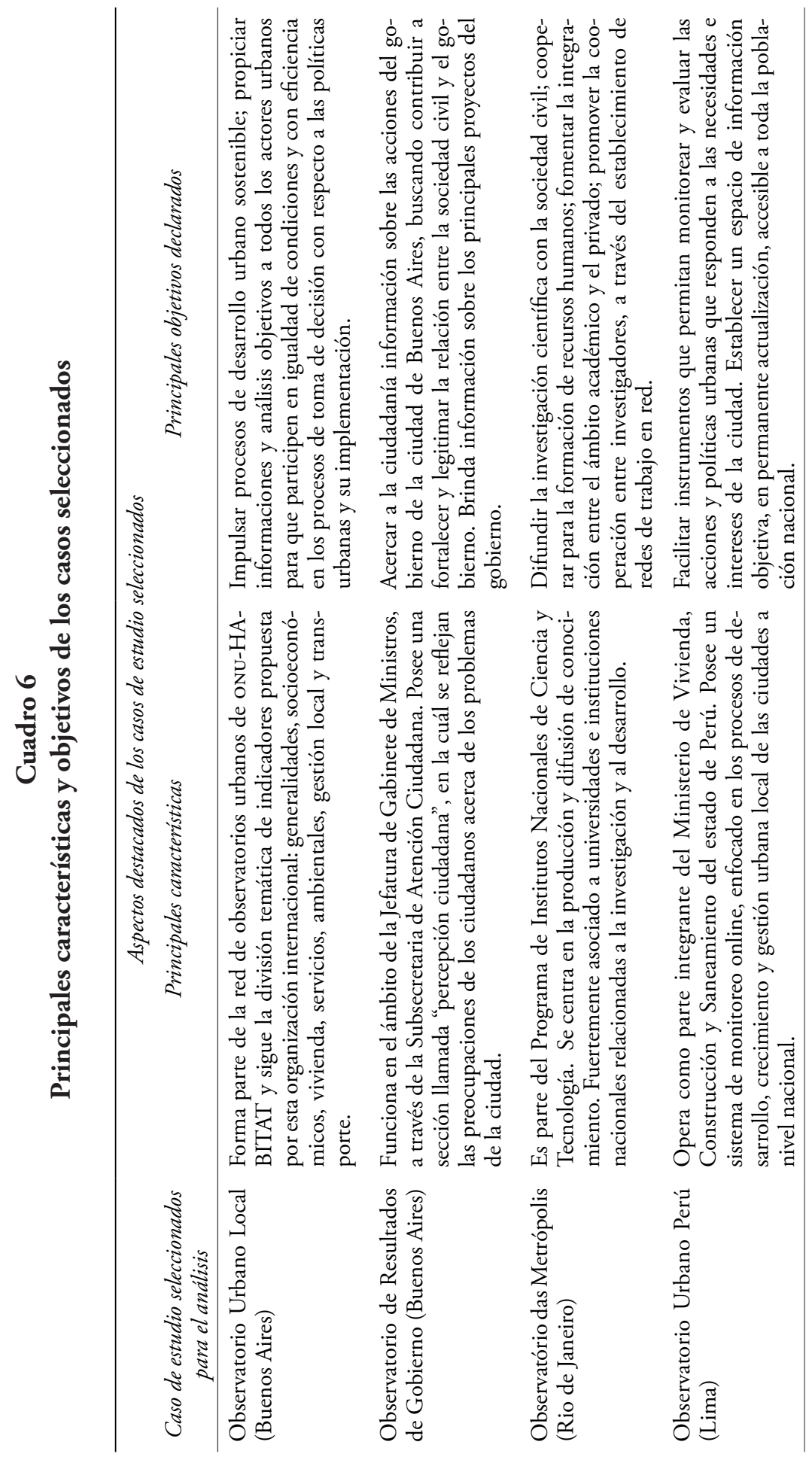




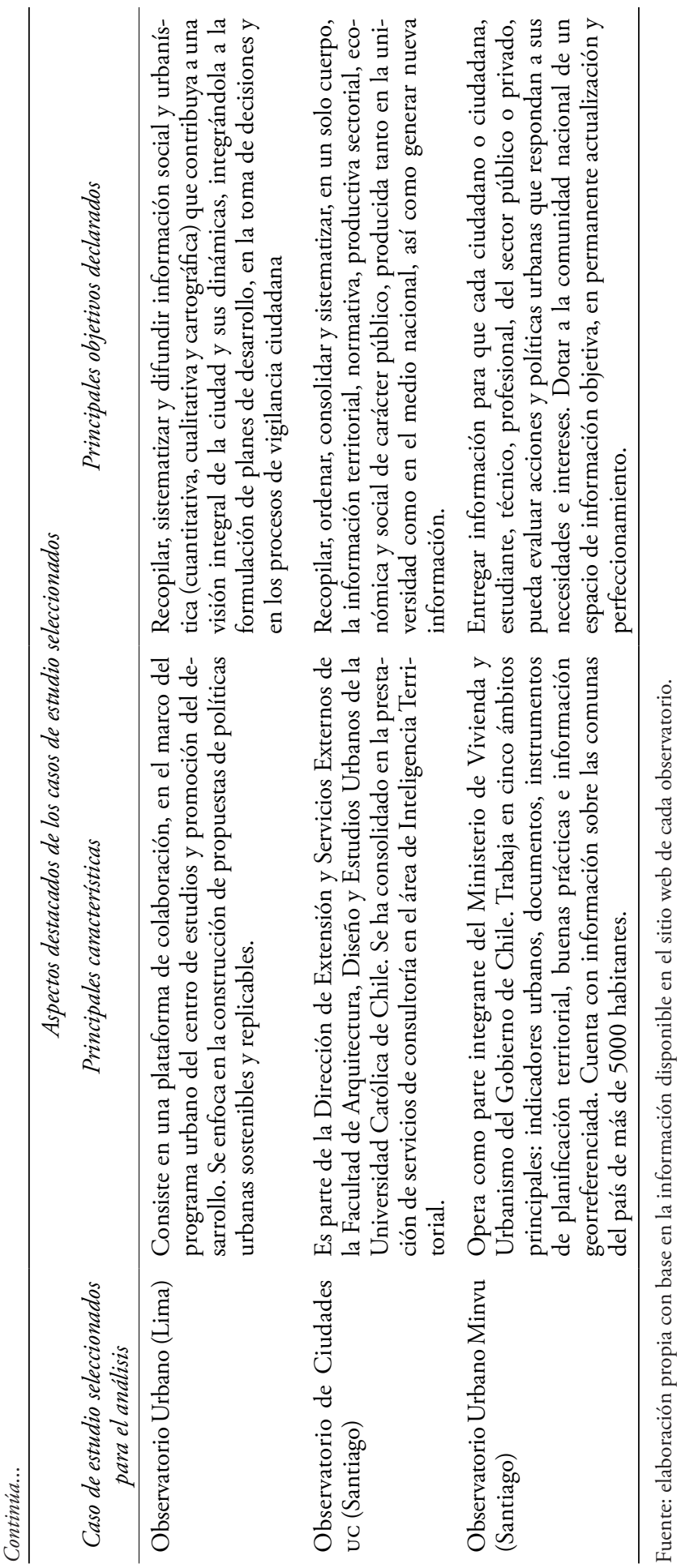




\section{Cuadro 7}

\section{Resultados de la aplicación de los criterios de participación a los casos seleccionados}

\begin{tabular}{|c|c|c|c|c|c|c|c|c|c|c|c|}
\hline \multirow[t]{2}{*}{ Criterio } & \multicolumn{3}{|c|}{ Nivel 1-Acceso } & \multicolumn{4}{|c|}{ Nivel 2-Consulta } & \multicolumn{4}{|c|}{ Nivel 3-Interacción } \\
\hline & $A$ & $B$ & $C$ & $D$ & $E$ & $F$ & $G$ & $H$ & $I$ & $J$ & $K$ \\
\hline $\begin{array}{l}\text { Observatorio Urbano } \\
\text { Local (Buenos Aires) }\end{array}$ & 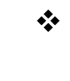 & & 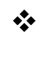 & & & & & & & & \\
\hline $\begin{array}{l}\text { Observatorio de Resultados } \\
\text { de Gobierno (Buenos Aires) }\end{array}$ & $*$ & $\nLeftarrow$ & 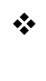 & & & & & $*$ & & & \\
\hline $\begin{array}{l}\text { Observatório das Metrópolis } \\
\text { (Rio de Janeiro) }\end{array}$ & $*$ & & 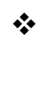 & & $\star$ & & & & & $\nLeftarrow$ & \\
\hline $\begin{array}{l}\text { Observatorio Urbano Perú } \\
\text { (Lima) }\end{array}$ & $*$ & & & & $\star$ & 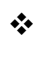 & & & & & \\
\hline Observatorio Urbano (Lima) & $\nLeftarrow$ & & $\nLeftarrow$ & & $*$ & $\nLeftarrow$ & $\star$ & & & & \\
\hline $\begin{array}{l}\text { Observatorio de Ciudades UC } \\
\text { (Santiago) }\end{array}$ & 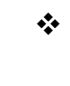 & & 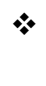 & & $*$ & & & & & & \\
\hline $\begin{array}{l}\text { Observatorio Urbano Minvu } \\
\text { (Santiago) }\end{array}$ & 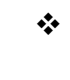 & & 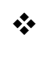 & & $*$ & & & & & & \\
\hline
\end{tabular}

Fuente: Carvalho (2013).

En este sentido, es necesario tener precaución antes de afirmar que este observatorio posibilita una participación fuerte, ya que si bien promueve la educación y la capacitación, aunque a veces indirectamente, lo hace para una porción específica de la población, la cual no se encuentra en el lado marginal por la brecha digital. De esta forma, si bien logra generar conocimiento valioso acerca de varias urbes brasileñas y colaborar para una mejor planificación de ellas, formando profesionales e investigadores capacitados en la temática territorial, no combate en línea de frente las dificultades impuestas por la brecha. No existe en el sitio web, por ejemplo, ningún glosario con términos asociados al territorio o a los estudios urbanos, que podrían ayudar aquellas personas que no llegan a la página con conocimientos previos más profundos acerca de la temática. Tampoco hay una sección de ayuda, que podría contar con las respuestas a las preguntas más frecuentes, facilitando la navegación y comprensión para el usuario.

En efecto, en sólo uno de los casos se encontró una sección dedicada al vocabulario específico que se ocupa para tratar la temática del territorio, pero aún así de forma incipiente. Fue el caso del Observatorio Urbano Local, dedicado específicamente a la metrópolis de Buenos Aires y directamente vinculado al programa de la ONU-Habitat de observatorios urbanos. 
Sin embargo, este observatorio no alcanza a superar el nivel 1 de participación, juntamente con el otro caso relativo a la ciudad de Buenos Aires. En alguna medida esto se debe a la falta de leyes de transparencia en este país, ya que la garantía de transparencia es esencial para el acceso a información confiable y verdaderamente informativa. De hecho, el Observatorio de Resultados de Gobierno llega a cumplir con un criterio de nivel 3, pero como no cumple con ninguno del nivel dos y los criterios son graduales, no siendo posible saltarse un paso, se queda en el nivel 1.

Acerca de los otros cuatro casos, que se encuentran en el nivel 2, llama la atención que sólo uno cumple con el criterio B (Observatorio Urbano Minvu). Más que eso, de todos los casos seleccionados sólo dos cuentan con herramientas para la visualización interactiva de la información territorial (el otro es el Observatorio de Resultados de Gobierno), un recurso que es altamente atractivo (incentivando el uso del sistema), que permite comparaciones, interacciones y que puede ser copiosamente didáctico. Además, si no fuera por el criterio $\mathrm{E}$, relativo al respaldo por una ley nacional de transparencia solamente dos alcanzarían el segundo nivel, ambos de Perú. Esto que indica que su posición como de nivel 2 es bastante débil, ya que depende de un criterio que es externo al caso en sí.

\section{Conclusiones}

Los observatorios urbanos deben, pueden y tienen que hacer más que sólo observar; sin embargo, con respecto a los que se analizaron en esta investigación no se puede decir que actualmente hacen mucho más que eso. Los resultados de este estudio ponen en evidencia el hecho de que los canales de participación establecidos por estos casos son, esencialmente, con un carácter y propósito informativos, promoviendo una participación a lo máximo simbólica (excepción, argumentable y posiblemente cuestionable por lo discutido anteriormente, al caso del Observatorio de las Metrópolis de Brasil) y, quizás incluso, la no-participación. Considerando que estos casos habían pasado una criba de selección bastante rigurosa -en relación a su compatibilidad con (i) los objetivos de la Cumbre Mundial sobre la Sociedad de la Información, y con (ii) aquellos de la Agenda 21; (iii) su vinculación con herramientas de planificación territorial; y (iv) con la existencia de un carácter transversal-territorial en sus enfoques- se esperaba que presentaran mejores posibilidades en cuanto a los niveles de participación que viabilizan, que fueran más proactivos, o bien, menos secundarios en su rol en procesos de planificación de estas metrópolis.

Vale destacar que a pesar de que el criterio relativo al uso de las Tig ha sido empleado en el proceso de selección de casos de estudio, el uso que se hace de estas tecnologías es aún muy incipiente y embrionario en el 
caso de los observatorios seleccionados. A pesar del gran potencial de las mismas para entregar información espacial a los ciudadanos-generando, por ejemplo, mapas interactivos georreferenciados con capas de información variadas o la visualización de posibles escenarios futuros relacionados a resultados de proyectos de planificación todavía en planta-, su uso en las plataformas web analizadas es bastante sencillo, más bien, banal desde una perspectiva territorial avanzada (toma de decisiones), muchas veces reducido a cartografía digitalizada.

Sin embargo, esto no significa que estos casos estén estancados en esta condición. De hecho es considerable su potencial, por ejemplo, como focos de ideas o think tanks, promoviendo la participación de la ciudadanía en los procesos de toma de decisión, particularmente en aquellos relacionados con la planificación, ya que estos, por su carácter transversal e integrador, vinculan diversas temáticas alrededor de una misma decisión espacial, sea en un ámbito urbano o metropolitano.

Si los observatorios llegan a implementar Sistemas de Información Territorial (Ganapati, 2011), Sistemas de Suporte a la Toma de Decisión con Geo-visualización (Bailey y Grossardt, 2009) o Sistemas de Procesamiento Espacial Analítico Online (McHugh et al., 2009), por ejemplo, ellos podrían entregar información sobre diversas temáticas e incluso utilizar los mecanismos de bidireccionalidad de flujos de información para recolectar datos, generando instancias en donde individuos y comunidades pudieran hacer comentarios, denuncias y demandas referidas en el espacio urbano. En este sentido, para hacer más que sólo observar, uno de los caminos posibles para los observatorios es la incorporación ciudadana en los procesos de gestión y producción de información referida al espacio geográfico.

Otra circunstancia a valorar es la de establecer un diálogo continuo y colaborativo entre estos observatorios, como opción estratégica, ya que posibilitaría, entre otras cosas, el intercambio de buenas prácticas y la replicabilidad de iniciativas exitosas. Esto se podría llevar a cabo a través de redes de cooperación descentralizada, haciendo uso, incluso, de redes ya establecidas, por ejemplo, la de las Mercociudades $^{2}$ o, idealmente la red de observatorios de la un-Habitat. Vale destacar que en esta red, uno de los objetivos centrales es el de compartir informaciones, conocimientos y experiencias, lo que podría ayudar a fortalecer el rol de cada uno de los observatorios participantes (UN-Habitat, 2014). Además, como establecen criterios comunes para la formulación de indicadores, se podría facilitar el proceso de identificar buenas prácticas y promover, a través de

\footnotetext{
${ }^{2}$ En efecto, la gran mayoría de las ciudades y metrópolis de los casos seleccionados para la etapa final ya son parte de esta red, en la cual se promueve y profundizan lazos de cooperación descentralizada como estrategia de desarrollo e internacionalización por parte de las municipalidades.
} 
acuerdos de cooperación internacional, por ejemplo, la replicación y adaptación de estas prácticas en otras ciudades. Para el caso específico de las metrópolis latinoamericanas, que comparten una serie de características y dinámicas como vulnerabilidad de los asentamientos informales, la creciente violencia urbana y la contaminación ambiental, fortalecer una red de observatorios urbanos es estratégico para el intercambio de experiencias y para la promoción de mayores niveles de calidad de vida urbana.

Para el fortalecimiento de los observatorios también es importante que ellos promuevan más prácticas educativas, paralelas al espacio digital en el cual operan sus páginas web, disminuyendo las brechas digitales que dificultan el acceso e incrementando e incentivando el uso de las mismas. Talleres de capacitación, reuniones presenciales e incluso clases son actividades que promueven el fortalecimiento del tejido social, esencial para un sentido de comunidad y para una participación comprometida. De esta forma, se trata más que de sólo posibilitar la participación, también de incentivarla y promoverla. Es indispensable que los ciudadanos sepan qué están viendo y de qué se está hablando, qué significan ciertos conceptos y qué consecuencias (externalidades espaciales) puede tener una propuesta en sus vidas. No basta con que un documento sea accesible, ${ }^{3}$ sino que su contenido y significado tiene que ser comprensible (Unesco, 2005).

Este estudio ha avanzado en la comprensión del rol que tienen estos observatorios urbanos actualmente, específicamente, en el contexto latinoamericano gracias al desarrollo de criterios para una evaluación cualitativa. Su principal contribución adviene de la construcción y aplicación de una metodología sencilla y operativa, diseñada específicamente para evaluar la contribución de estas entidades en lo que se refiere a las posibilidades que generan para la incorporación ciudadana en procesos de planificación metropolitana a través de su presencia en la internet; sin embargo, hay que tener en cuenta que estos resultados son derivados de un grupo acotado de observatorios urbanos seleccionados. Para dar continuidad a esta línea de investigación, en el futuro será importante abarcar más observatorios, enriqueciendo el análisis comparativo respecto a su diseño operativo, carácter civil o gubernamental, flujo de información o pertenencia a una red.

En sintonía con el argumento de Carver (2003), se considera de vital importancia la capacidad de visualizar espacialmente, o mejor dicho, territorialmente, la visión de futuro para que se pueda llevar a cabo una

\footnotetext{
${ }^{3}$ Acerca del punto relativo a, "documento estar disponible", es importante tener en cuenta que la legislación de cada país juega un rol extremadamente importante, específicamente debido a su ley de transparencia (o falta de ella, como es el caso de Argentina). En el caso de los países sudamericanos abarcados en esta última selección de casos, se destaca Chile por el funcionamiento de su ley de transparencia, "ante todo porque existe un ente autónomo eficiente que dirime las denegaciones de información y por sus niveles de transparencia activa” (Huerta, 2013).
} 
planificación sustentable. Los observatorios podrían ganar preeminencia en este frente, cooperando para una participación ciudadana más fuerte en la planificación metropolitana, sacando partido de las posibilidades de recolección, sistematización y presentación de datos e informaciones territoriales que las TIG ofrecen para el futuro de la sociedad en red.

\section{Bibliografía}

Álvarez-Lobato, José Antonio, Tania Chávez y Carlos Garrocho (2007), "El observatorio metropolitano de Toluca: lecciones, propuestas y desafíos", Economia, Sociedad y Territorio, VII (25), El Colegio Mexiquense, Zinacantepec, pp. 157-214.

Arnstein, Sherry (1969), "A ladder of citizen participation", Journal of the American Planning Association, 35 (4), American Planning Asociation,Taylor \& Francis Online, Washington, pp. 216-224.

Bailey, Keiron y Ted Grossardt (2009), "Toward structured public involvement: justice, geography and collaborative geospatial/geovisual decision support systems", Annals of the Association of American Geographers, 100 (1), Routledge, Philadelphia, pp. 57-86.

вм (Banco Mundial) (2014), ICT 4 GOV: Empowering citizens to demand better governance, Banco Mundial, Washington, <http://ict4gov. net/>, 20 de septiembre de 2013.

Carsjens, Gerrit-Jan y Arend Ligtenberg (2007), "A GIS-based support tool for sustainable spatial planning in metropolitan areas", Landscape and Urban Planning, 80 (1-2), Elsevier, Cambridge, pp. 72-83.

Carvalho, Juliana (2013), "Conexiones virtuales, brechas sociales, prácticas gubernamentales: Estudio exploratorio acerca de las posibilidades de incorporación ciudadana en procesos de planificación territorial a través del uso de tecnologías de información geoespacial", tesis de grado de magíster, Instituto de Estudios Urbanos y Territoriales, Pontificia Universidad Católica de Chile, Santiago.

Carver, Steve (2003), "The future of participatory approaches using geographic information: developing a research agenda for the 21th Century", URISA Journal, 15 (APA I), Des Plaines, pp. 61-71. 
Castells, Manuel (1995), La ciudad informacional. Tecnologias de la información, reestructuración económica y el proceso urbano-regional, Alianza, Madrid.

Cicolella, Pablo (2006), "Metrópolis latinoamericanas: territorios subregulados, ¡espacios del capital?”, en Adrián Guillermo (coord.), Las grandes aglomeraciones y su periferia regional. Experiencias en Latinoamérica y España, UnAM, México, pp. 305-324.

Davoudi, Simin e Ian Strange, (2009), Conceptions of space and place in strategic spatial planning, Routledge, Londres.

Dennis, Samuel (2006), "Prospects for qualitative GIS at the intersection of youth development and participatory urban planning", Environment and Planning A, 38 (11), Pion Publication, Londres, pp. 2039-2054.

Duncan, Sally y Denise Lach (2006), "GIS technology in natural resource management: process as a tool of change", Cartographica: The International Journal for Geographic Information and Geovisualization, 41 (3), University of Toronto Press, Toronto, pp. 201-215.

Elwood, Sarah (2006), "Critical issues in participatory GIS: deconstructions, reconstructions, and new research directions", Transactions in GIS, 10 (5), Blackwell Publishing, Oxford, pp. 693-708.

Ferreira, Andreia Cristina, Lígia Torres Silva y Rui Antonio Rodrigues Ramos (2012), "Urban observatories, tools for monitoring cities”, en Ramos, Rui Antonio Rodrigues, Inga Straupe y Thomas Panagopoulos (eds.), Recent Researches in Environment, Energy Systems and Sustainability, WSEAS Press, Faro, Portugal, pp. 259-264.

Freire, Juan y Daniel Villar Onrubia, (2010), "Prácticas cartográficas cotidianas en la cultura digital”, Razón y Palabra, 73, Proyecto Internet del itesm, Campus Estado de México, Atizapán de Zaragoza, pp. 1-14, <www.razonypalabra.org.mx>, 22 de septiembre de 2012.

Fusero, Paolo (2009), E-city: digital networks and cities of the future, List Laboratorio, Roma. 
Ganapati, Sukumar (2011), "Uses of public participation geographic information systems applications in E-Government", Public Administration Review, 71 (3), Wiley-Blackwell Publishing, Indianapolis, pp. 425-434.

González, Ainhoa, Alan Gilmer, Ronan Foley, John Sweeney y John Fry (2008), "Technology-aided participative methods in environmental assessment: An international perspective", Computers, Environment and Urban Systems, 32 (4), Elsevier, Cambridge, pp. 303-316.

Graham, Stephen (2000), "Las telecomunicaciones y el futuro de las ciudades derribando mitos", EURE, 26 (77), Editorial de la Pontificia Universidad Católica de Chile, Santiago, pp. 5-23.

Hansen, Henning Sten y David Prosperi (2005), “Citizen participation and internet GIS-Some recent advances", Computers, Environment and Urban Systems, 29 (6), Elsevier, Cambridge, pp. 617-629.

Hanzl, Malgorzata (2007), "Information techonology as a tool for public participation in urban planning: a review of experiments and potentials", Design Studies, 28, Elsevier, Cambridge, pp. 289-307.

Haque, Akhlaque (2001), "GIS, public service, and the issue of democratic governance", Public Administration Review, 61 (3), Blackwell Publishing, Oxford, pp. 259-265.

Hendriks, Paul y Dirk Vriens (2000), "From geographical information systems to spatial group decision support systems: a complex itinerary", Geographical and Environmental Modelling, 4 (1), Routledge, Philadelphia, pp. 83-104.

Hernández-Moreno, Silverio (2009), "Current technologies applied to urban sustainable development", Theoretical and Empirical Researches in Urban Management, 4 (13), Research Centre in Public Administration and Public Services Press, Bucarest, pp. 125-140.

Huerta, Helmuth (2013), "Información ambiental para la democracia: comparación de la situación de América Latina y de Chile con la Convención de AARHUS", tesis para optar al título de magíster en gestión y planificación ambiental, Programa Interfacultades, Universidad de Chile, Santiago. 
ITU (International Telecommunication Union) (2006), "Agenda de Túnez para la Sociedad de la Información”, ITU, Túnez, <https://www. itu.int/wsis/docs2/tunis/off/6rev1-es.html>, 3 de mayo de 2013.

Kearns, Faith, Maggi Kelly y Karin Tuxen (2003), "Everything happens somewhere: using webGIS as a tool for sustainable natural resource management", Frontiers in Ecology and the Environment, 1 (10), Ecological Society of America Press, Washington, pp. 541-548.

Kingston, Richard, Steve Carver, Andrew Evans y Ian Turton (2000), "Web-based public participation geographical information systems: an aid to local environmental decision-making", Computers, Environment and Urban Systems, 24 (2), Elsevier, Cambridge, pp. 109-125.

Mansourian, Ali, Mohammad Taleai y Ali Fasihi (2011), "A web-based spatial decision support system to enhance public participation in urban planning processes", Journal of Spatial Science, 56 (2), Routledge, Philadelphia, pp. 269-282.

McCall, Michael y Christine Dunn (2012), "Geo-information tools for participatory spatial planning: Fufilling the criteria of 'good' governance?", Geofrorum, 43, Elsevier, Londres, pp. 81-94.

McHugh, Rosemarie, Stéphane Roche y Yvan Bédard (2009), "Towards a SOLAP-based public participation GIS", Journal of Environmental Management, 90 (6), Elsevier, Cambridge, pp. 2041-2054.

Nogué, Joan, Laura Puigbert, Pere Sala y Gemma Bretcha (2010), "Paisatge i participació ciutadana", Observatori del Paisatge Generalitat de Catalunya, Barcelona, <http://www.catpaisatge.net/esp/ documentacio_doc_1.php>, 8 de agosto de 2012.

Orellana, Arturo (2013), "Gobiernos metropolitanos para Chile: la necesidad versus la factibilidad”, Temas de la Agenda Pública, 8 (63), Centro de Políticas Públicas, Pontificia Universidad Católica de Chile, Santiago, pp. 1-16.

Portes, Alejandro, Bryan Roberts y Alejandro Grimson (2008), Ciudades latinoamericanas un análisis comparativo en el umbral del nuevo siglo, Universidad Autónoma de Zacatecas-Miguel Ángel Porrúa, México. 
Repetti, Alexandre y Roland Prélaz-Droux (2003), "An Urban Monitor as support for a participative management of developing cities", Habitat International, 27 (4), Elsevier, Cambridge, pp. 653-667.

Rojas-Caldelas, Rosa y Eva Alicia Corona-Zambrano (2008), “Urban observatories opportunities for environmental monitoring: solid wastes", Waste Management, 28 (1), Elsevier, Cambridge, pp. $40-44$.

Song, Weidong y Guibo Sun (2010), “The role of mobile volunteered information in urban management", Trabajo publicado en la $18^{\mathrm{a}}$ Conferencia Internacional de Geomática, Fuxin, China, 18-20 de junio.

UN (United Nations) (2001), Agenda 21, United Nations, New York, $<$ http://www.un.org/spanish/esa/sustdev/agenda21/>, 03 de mayo de 2013.

UNESCO (2005), "Hacia las sociedades del conocimiento”, unEsCo, Francia, <http://unesdoc.unesco.org/images/0014/001419/141908s. pdf>, 26 de enero de 2013

un-Habitat (2014), "Global Urban Observatory”, un-Habitat ProgramesUnited Nations, Nairobi, Kenya, <http://ww2.unhabitat.org/ programmes/guo/default.asp>, 20 de diciembre de 2013.

Valenzuela, Luis Miguel y Julio Alberto Soria (2011), "Observatorios territoriales y urbanos en Europa ¿entidades pasivas o instrumentos operativos para la planificación?”, Ciudad y territorio: estudios territoriales, 168, Ministerio de Fomento, Gobierno de España, Madrid, pp. 243-260.

Vajjhala, Shalini (2006), “'Ground Truthing’ policy: using participatory map-making to connect citizens and decision makers", Resources, 162, Resources for the Future Organization, Basel, Suiza, pp. 14-18.

Yeh, Anthony Gar-On (2008), GIS as a planning support system for the planning of harmonious cities, UN-Habitat, Nairobi. 


\section{Páginas de los observatorios}

\section{Argentina:}

Mapa Interactivo de Buenos Aires, <http://mapa.buenosaires.gob.ar/>, 25 de agosto de 2013.

Observatorio Urbano Local, <http://www.oulbam.com.ar/>, 26 de agosto de 2013.

Observatorio de Resultados del Gobierno, <http://www.observatoriodegobierno.buenosaires.gob.a r/>, 26 de agosto de 2013.

\section{Brasil:}

Observatório do Milenio, <https://observatoriodomilenio.pbh.gov.br/>, 10 de julio de 2013.

Rio como vamos, <http://www.riocomovamos.org.br/portal/index.php>, 12 de julio de 2013.

Rede Nossa São Paulo, <http://www.nossasaopaulo.org.br/portal/>, 12 de julio de 2013.

Observatório das Metrópolis, <http://web.observatoriodasmetropoles. net/>, 10 de julio de 2013.

\section{Chile:}

CAS-Climate Adaptation Santiago, <http://www.ufz.de/climate-adaptation-santiago/>, 3 de julio de 2013.

Suma Verde, <http://www.sumaverde.cl/>, 4 de julio de 2013.

Observatorio de Ciudades UC, <http://www.ocuc.cl/>, 2 de julio de 2013.

Observatorio Urbano Minvu, <http://www.observatoriourbano.cl/index. asp>, 4 de julio de 2013 .

\section{Colombia:}

SIGUE, <http://web.acueducto.com.co/sigueweb/index.jsp>, 15 de agosto de 2013. 
Red de Ciudades cómo vamos, <http://www.bogotacomovamos.org/ acerca-de/red-de-ciudades/>, 20 de agosto de 2013.

\section{Perú:}

Observatorio Urbano, <http://www.observatoriourbano.org.pe/>, 3 de agosto de 2013.

Observatorio Urbano Perú, <http://febe.vivienda.gob.pe:8080/OBSERVATORIO/>, 3 de agosto de 2013.

\section{Uruguay:}

Observatorio de Montevideo, <http://www.montevideo.gub.uy/ciudad/ aplicacion/observatorio-de-montevideo >, 10 de agosto de 2013.

Recibido: 19 de febrero de 2014. Reenviado: 30 de junio de 2014. Aceptado: 13 de agosto de 2014.

Luis Miguel Valenzuela-Montes. Español. Doctor en geografía por la Universidad de Granada (2000), España. Actualmente es profesor titular del Departamento de Urbanística y Ordenación del Territorio, y coordinador del máster en urbanismo (Universidad de Granada). Sus principales líneas de investigación se enfocan en el diseño de escenarios de desarrollo territorial, la evaluación de la planificación y la integración urbana en torno a corredores de transporte. Entre sus últimas publicaciones destacan, en coautoría: "Paesaggio agrario nei piani locali e d'area vasta in Spagna”, Urbanistica. INU Edizioni, núm. 150-151, Istituto Nazionale di Urbanistica, Roma, pp. 52-56 (2013); "A method for the evaluation of metropolitan planning. Application to the context in Spain", European Planning Studies, 21 (6), Taylor \& Francis Group-Routledge, Londres, pp. 944-966 (2013); “Enfoque metodológico para la valoración de escenarios de movilidad urbana frente al cambio climático", ACE: Architecture, City and Environment=Arquitectura, Ciudad y Entorno [en línea], 7 (19), Centre de Política del Sòl i Valoracions, Universitat Politècnica de Catalunya, Barcelona, pp.111-128 (2012); "Observatorios territoriales y urbanos en Europa: ¡entidades pasivas o instrumentos operativos para la planificación?”, Ciudad y Territorio. Estudios Territoriales, XLIII (168), Ministerio de Fomento, Gobierno de España, Madrid, pp. 243-260 (2011). 
Juliana Carvalho-Cortes Silva. Brasileña. Es magíster en asentamientos humanos y medio ambiente por la Pontificia Universidad Católica de Chile. Actualmente es asistente de investigación en el Centro uc de Cambio Global y en el Instituto de Estudios Urbanos y Territoriales de la Facultad de Arquitectura, Diseńo y Estudios Urbanos de la misma universidad. Sus líneas de investigación actuales son la participación ciudadana en procesos de toma de decisión y la calidad de vida urbana. 